Article

\title{
Interaction between Antifungal Isoxazolo[3,4-b]Pyridin 3(1H)-One Derivatives and Human Serum Proteins Analyzed with Biomimetic Chromatography and QSAR Approach
}

\author{
Krzesimir Ciura ${ }^{1, *}$, Joanna Fedorowicz ${ }^{2}$ (D), Hanna Kapica ${ }^{1}$, Monika Pastewska ${ }^{1}$, Wiesław Sawicki ${ }^{1}$ and \\ Jarosław Sączewski ${ }^{3}$ (D) \\ 1 Department of Physical Chemistry, Medical University of Gdańsk, Al. Gen. Hallera 107, \\ 80-416 Gdańsk, Poland; hanna.kapica@gumed.edu.pl (H.K.); monika-pastewska@gumed.edu.pl (M.P.); \\ wsawicki@gumed.edu.pl (W.S.) \\ 2 Department of Chemical Technology of Drugs, Faculty of Pharmacy, Medical University of Gdańsk, Al. Gen. \\ J. Hallera 107, 80-416 Gdańsk, Poland; joanna.fedorowicz@gumed.edu.pl \\ 3 Department of Organic Chemistry, Faculty of Pharmacy, Medical University of Gdańsk, \\ Al. Gen. J. Hallera 107, 80-416 Gdańsk, Poland; jaroslaw.saczewski@gumed.edu.pl \\ * Correspondence: krzesimir.ciura@gumed.edu.pl
}

check for

updates

Citation: Ciura, K.; Fedorowicz, J.; Kapica, H.; Pastewska, M.; Sawicki, W.; Saczewski, J. Interaction between Antifungal Isoxazolo[3,4-b]Pyridin 3(1H)-One Derivatives and Human Serum Proteins Analyzed with Biomimetic Chromatography and QSAR Approach. Processes 2021, 9, 512. https://doi.org/10.3390/ pr9030512

Academic Editor: Alina Pyka-Pają

Received: 10 February 2021

Accepted: 9 March 2021

Published: 12 March 2021

Publisher's Note: MDPI stays neutral with regard to jurisdictional claims in published maps and institutional affiliations.

Copyright: (C) 2021 by the authors. Licensee MDPI, Basel, Switzerland. This article is an open access article distributed under the terms and conditions of the Creative Commons Attribution (CC BY) license (https:// creativecommons.org/licenses/by/ $4.0 /)$
Abstract: The development of effective, nontoxic antifungal agents is one of the most important challenges for medicinal chemistry. A series of isoxazolo [3,4-b]pyridine-3(1H)-one derivatives previously synthesized in our laboratory demonstrated promising antifungal properties. The main goal of this study was to investigate their retention behavior in a human serum proteins-high-performance liquid chromatography (HSA-HPLC) system and explore the molecular mechanism of HSA-isoxazolone interactions using a quantitative structure-retention relationship (QSRR) approach. In order to realize this goal, multiple linear regression (MLR) modeling has been performed. The proposed QSRR models presented correlation between experimentally determined lipophilicity and computational theoretical molecular descriptors derived from Dragon 7.0 (Talete, Milan, Italy) software on the affinity of isoxazolones to HSA. The calculated plasma protein binding (PreADMET software) as well as chromatographic lipophilicity $\left(\log k_{\mathrm{W}}\right)$ and phospholipophilicity $\left(\mathrm{CHI}_{\mathrm{IAM}}\right)$ parameters were statistically evaluated in relation to the determined experimental HAS affinities $\left(\log k_{\mathrm{HSA}}\right)$. The proposed model met the Tropsha et al. criteria $R^{2}>0.6$ and $Q^{2}>0.5$ These results indicate that the obtained model can be useful in the prediction of an affinity to HSA for isoxazolone derivatives and they can be considered as an attractive alternative to HSA-HPLC experiments.

Keywords: human serum albumin (HSA); biomimetic chromatography; isoxazolone; QSRR analysis

\section{Introduction}

Fungal infections constitute a significant threat to the public healthcare sector [1-3]. The development of new antifungal agents is definitely more complex and complicated than the elaboration of antibacterial substances due to the eukaryotic nature of the fungi cells [4]. Therefore, only a few class of chemicals, such as polyenes, azoles, echinocandins, allylamines, and flucytosine, are used in routine medical practice [3]. Another problem of treatment of fungal infections is systematic increase in resistance to available drugs. It should be highlighted that resistance was not only reported in cases of well-known drugs such as fluconazole but some cases also for recently introduced echinocandin $[3,5,6]$. At the same time, the use of numerous effective antifungal drugs including amphotericin B is limited by their unfavorable safety profiles [7]. Consequently, the search of more effective and safe antifungal molecules is a primary concern in the drug discovery pipeline.

The plasma protein binding (PPB) affinity is usually expressed in the literature as a percentage of bound drug molecules [8]. Affinity to plasma proteins should be taken under consideration in research projects aimed at design and assessment of drug candidates 
since PPB determines drug distribution, half-life, and clearance. Additionally only free drug fraction can penetrate through biological membranes [9]. As a result, only unbound molecules may reach the intended receptor and exert a pharmacological response. Furthermore, the safety of pharmacotherapy is also linked with PPB. Generally a molecule with high PPB affinity ( $>95 \%$ ) cause several adverse effects such as low clearance and brain penetration, as well as significant risk of drug-drug interaction [9]. For this reason, drug-plasma protein interactions are very important from pharmacokinetic, toxicological and pharmacological point of view. Human serum albumin (HSA) is the predominant protein in blood plasma which constitutes approximately $50 \%$ of the proteins present in the plasma. HSA is responsible for fatty acids transport and balance of the colloidal osmotic blood pressure. This protein is a single-chain, non-glycosylated polypeptide containing 585 amino acids composed of three homologous domains: I, II, and III. Hence, in HSA structures three main drug binding subdomains exist: sites IIA and IIIA, as well as site IB [10].

A series of isoxazolo[3,4-b]pyridine-3(1H)-one derivatives, previously synthesized in our laboratory, proved noticeable antifungal activity against Candida parapsilosis (minimum inhibitory concentration (MIC) $<6.2 \mu \mathrm{g} / \mathrm{mL}$ ) [11-13]. These structures have been characterized in terms of lipophilicity using chromatographic and theoretical approaches [14,15]. Additionally, immobilized artificial membrane (IAM) chromatography experiments has been performed to examine the interactions that occur between biological membranes and the synthesized isoxazolone derivatives [14]. Although the above-mentioned methods are helpful in prediction of biological properties, they do not fully describe moleculeprotein interactions, which may be highly specific. Consequently, the aim of this work was to investigate retention behavior of isoxazolo[3,4-b]pyridine-3(1H)-one derivatives in HSA-HPLC system and to explore molecular mechanism of HSA-isoxazolone interactions using quantitative structure-retention relationship (QSRR) approach. The proposed QSRR model links affinity of the investigated isoxazolones to HSA with experimentally determined physicochemical properties such as lipophilicity and phospholipophilicity as well as computational theoretical molecular descriptors. PPB of the target compounds was also estimated using PreADMET software since computational and experimental approaches should be complementary in characterization of HSA-isoxazolones interplay. To the best of our knowledge, the present study is the first report in the scientific literature that pertain to molecular modeling of isoxazolone-HSA interactions. Moreover, the results allow for the selection of promising structures for further studies. Molecules that show the lowest affinity to HSA should be chosen, since they have the best safety profiles among the surveyed group of solutes.

\section{Materials and Methods}

\subsection{Chemical Reagents}

Dimethyl sulfoxide (DMSO) used to dissolve analytes was purchased from $\mathrm{POCH}$ (Gliwice, Poland). 2-Propanol HPLC grade for liquid chromatography, sodium phosphate dibasic dihydrate and sodium phosphate monobasic monohydrate were obtained from Sigma-Aldrich (Steinheim, Germany). Water was purified by using Millipore Direct-Q 3 UV Water Purification System (Millipore Corporation, Bedford, MA, USA), prior to mobile phase preparation. The mobile phase was mixed and degassed just before the analysis.

\subsection{Analytes}

The examined pyrido- and quinolino-isoxazolones were synthesized and purified according to the procedures described in details elsewhere [11-13]. The 2D structures of the investigated molecules are presented in Figure 1, whereas the corresponding chemical names are listed Supplementary Table S1. Before HPLC analyses all the studied compounds were dissolved in DMSO to obtain a concentration of $1 \mathrm{mg} / \mathrm{mL}$ and stored at $2-8{ }^{\circ} \mathrm{C}$. The stock solutions of the solutes were diluted to attain concentrations of $100 \mu \mathrm{g} / \mathrm{mL}$ priori to HPLC experiments. 
<smiles></smiles>

1: $R^{1}=R^{2}=R^{3}=R^{4}=R^{5}=H$

2: $R^{1}=B n ; R^{2}=R^{3}=R^{4}=R^{5}=H$

3: $R^{1}=M e ; R^{2}=R^{3}=R^{4}=R^{5}=H$

4: $R^{1}=B z ; R^{2}=R^{3}=R^{4}=R^{5}=H$

14: $R^{1}=M e ; R^{2}=H ; R^{3}=O M e ; R^{4}=R^{5}=H$

15: $R^{1}=M e ; R^{2}=H ; R^{3}=F ; R^{4}=R^{5}=H$

16: $R^{1}=M e ; R^{2}=H ; R^{3}=C l ; R^{4}=R^{5}=H$

17: $R^{1}=M e ; R^{2}=H ; R^{3}=R^{4}=M e ; R^{5}=H$

18: $R^{1}=H ; R^{2}=O M e ; R^{3}=R^{4}=H ; R^{5}=O M e$

19: $R^{1}=M e ; R^{2}=R^{3}=H ; R^{4}=M e ; R^{5}=H$

20: $R^{1}=M e ; R^{2}=O M e ; R^{3}=R^{4}=H ; R^{5}=O M e$

21: $R^{1}=M e ; R^{2}=H ; R^{3}=M e ; R^{4}=R^{5}=H$<smiles>[R]n1oc(=O)c2c(C)cc(C)nc21</smiles>

5: $\mathrm{R}^{1}=\mathrm{Bn}$

6: $R^{1}=A c$

7: $\mathrm{R}^{1}=\mathrm{Et}$

8: $R^{1}=B z$

9: $\mathrm{R}^{1}=\mathrm{Me}$

10: $\mathrm{R}^{1}=\mathrm{CH}_{2} \mathrm{C}_{6} \mathrm{H}_{3}-3,5-\mathrm{diOMe}$

11: $\mathrm{R}^{1}={ }^{n} \mathrm{Bu}$

12: $\mathrm{R}^{1}=n \mathrm{Pr}$

13: $\mathrm{R}^{1}=\mathrm{H}_{2} \mathrm{C}-\mathrm{C} \equiv \mathrm{CH}$

Figure 1. The investigated pyrido- and quinolino-isoxazolones.

\subsection{HSA-HPLC Analysis}

All the HSA-HPLC experiments were carried out using a Prominence-1 LC-2030C 3D HPLC system (Shimadzu, Japan) controlled by LabSolution system (version 5.90, Shimadzu, Japan) equipped with a Diode-Array Detection (DAD) detector and a HSA column $(0.4 \mathrm{~cm}$ $\times 10 \mathrm{~cm} \times 5 \mu \mathrm{m}$ particle size, Chiral Technologies Europe, Illkirch-Graffenstaden, France). The injected volume was $5 \mu \mathrm{L}$. The HSA-HPLC analyses were carried with an isocratic conditioning. The composition of mobile phase was as follows $10 \%$ of 2-propanol and $10 \mathrm{mM}$ of phosphoric buffer at $\mathrm{pH} 7.4$ with the flow rate of $0.9 \mathrm{~mL} / \mathrm{min}$. The temperature of chromatographic column was controlled and set to o $25.0^{\circ} \mathrm{C}$ while the analysis time was $15 \mathrm{~min}$. The retention times of target molecules are listed in Supplementary Table S2 whereas Figure 2 presents representative chromatograms. The $\log k_{\mathrm{HSA}}$ values have been calculated according to Equation (1):

$$
\log k_{\mathrm{HSA}}=\log \frac{t_{R}-t_{0}}{t_{0}}
$$

where $t_{R}$ and $t_{0}$ are the retention times of the analytes and DMSO, respectively. Hence, the latter was used as dead time marker (1.2701 $\mathrm{min})$. All the measurements were performed in triplicate and the values for the logarithm of the chromatographic retention factor of HSA column $\left(\log k_{\mathrm{HSA}}\right)$ have been calculated as the average of three replicates.
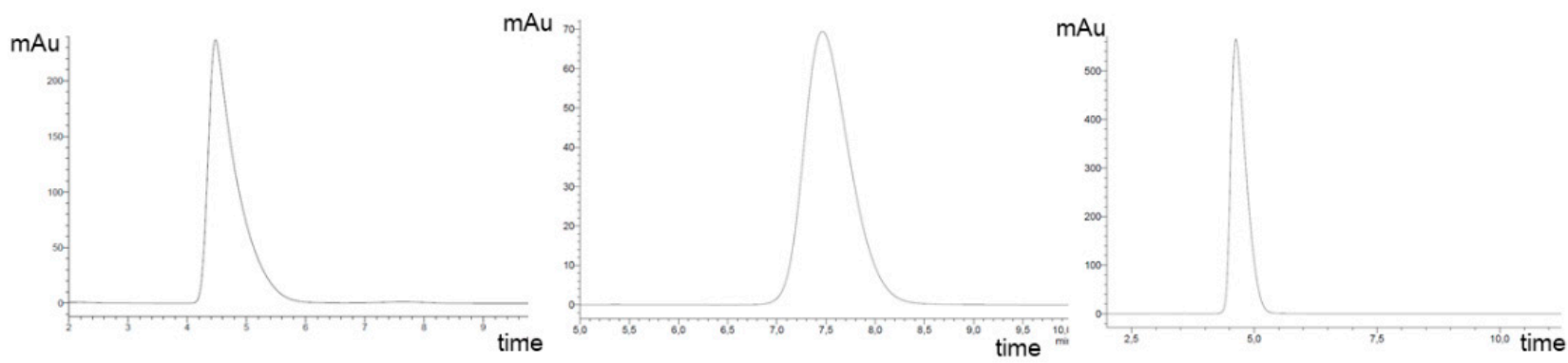

Figure 2. The representative chromatograms of compounds 1, 5, and 15. 


\subsection{QSRR Analysis}

The theoretical descriptors were calculated applying Dragon 7.0 (Talete, Milan, Italy) software and based on geometries obtained previously [15], i.e., structures optimized with Gaussian 16 package by means of density functional theory (DFT) at the PBE0/6$311 \mathrm{G}++(2 \mathrm{df}, 2 \mathrm{pd})$ level of theory [16] and the SMD solvation model [17]. Finally, 2581 theoretical descriptors have been calculated for each molecule. In the performed QSRR study, the predictor variable was the retention factor $\left(\log k_{\mathrm{HSA}}\right)$, whereas theoretical molecular descriptors as well as previously chromatographically determined lipophilicity $\left(\log k_{\mathrm{w}}\right)$ [14] and affinity to phospholipids $\left(\mathrm{CHI}_{\text {IAM }}\right)$ [14] served as independent variables. The calculation matrix was pre-edited prior to QSRR analysis. In the first step constant and near constant descriptors were eliminated. Hence, 499 best predictors were selected using the F-test algorithm implemented into Statistica software (Statistica 13.3, StatSoft, Tulsa, OK, USA). The calculations of QSRR models were performed using multiple linear regression (MLR) with a forward selection mode based on F criteria ( $\mathrm{F}>1.0$ ). QSRR models based only on the theoretical computational descriptors as well as linking both experimental and computational data are presented.

The coefficients of correlation $(R)$ and determination $\left(R^{2}\right)$, standard deviation, and the standard estimation error (s) were used as the bases for linearity testing of the regression plots. Additionally $Q^{2}$ were calculated with the leave-one-out validation approach and root-mean-squared error of cross-validation (RMSECV).

\subsection{In-Silico Calculation}

In-silico PPB\% values for the studied solutes have been obtained using PreADME software (https: / / preadmet.bmdrc.kr/ accessed on 2 September 2020). Input structures for PreADME calculations were generated on the basis of optimized structures as a set of mol files.

\section{Results and Discussion}

In order to determine HSA binding affinity of the studied isoxazolone derivatives biomimetic HPLC analysis has been performed. Direct methods for the assessment of PPB such as ultrafiltration and equilibrium dialysis are laborious, time consuming and require sophisticated analytical instrumentations. HPLC columns that comprise immobilized HSA were introduced for chiral chromatographic separations [18]. However, a good agreement between retention on HSA column and binding affinity to HSA proteins determined with equilibrium dialysis and ultrafiltration $[19,20]$ allowed for the application of the HSAHPLC approach for reliable HSA-xenobiotic binding affinity assessment. Additionally, this chromatographic approach has a significant advantage since it allows to estimate compound binding in very reproducible and robust manner regardless of changes in protein levels in the blood serum of individual patients.

The experimentally determined $\log k_{\mathrm{HSA}}$ parameters, calculated $\mathrm{PPB} \%$, chromatographically assessed lipophilicity $\left(\log k_{\mathrm{w}}\right)$, as well as affinity to phospholipids $\left(\mathrm{CHI}_{\mathrm{IAM}}\right)$ are listed in Table 1. The 2D structures and chemical names of the studied compounds and can be found in Supplementary Table S1. Among the tested molecules, the highest affinity to HSA showed compounds 16 and 17, i.e., 6-chloro-1-methyl and 1,6,7-trimethyl quinoline derivatives, respectively. These compounds displayed also high affinities to phospholipids and explicit lipophilic character $\left(\log k_{\mathrm{w}}>3.0\right)$. Oppositely, molecules 2, 6, 7, and 9 have proven very weak affinities to HSA. These compounds except for molecule 2 are based on pyridine skeleton. Hence, in general, chromatographic retentions determined with HSA column suggest that pyridine derivatives feature rather weak affinities to HSA. 
Table 1. Chromatographically determined $\log k_{\mathrm{HSA}}$ parameters, lipophilicity indexes $\left(\log \mathrm{k}_{\mathrm{W}}\right)$ and affinity to phospholipids $\left(\mathrm{CHI}_{\mathrm{IAM}}\right)$ along with calculated $\mathrm{PPB} \%$.

\begin{tabular}{ccccc}
\hline No & $\log k_{\mathbf{H S A}}$ & $\log \boldsymbol{k}_{\mathbf{w}}{ }^{*}$ & $\mathbf{C H I}_{\mathbf{I A M}}{ }^{*}$ & PPB $\boldsymbol{~}^{* *}$ \\
\hline 1 & 2.57 & 2.36 & nd & 83.78 \\
2 & 0.47 & 3.98 & 12.92 & 93.94 \\
3 & 1.78 & 2.87 & 24.85 & 85.62 \\
4 & 2.58 & 3.76 & nd & 92.81 \\
5 & 4.78 & 3.68 & 34.01 & 61.59 \\
6 & 0.56 & 2.53 & 14.91 & 42.95 \\
7 & 0.79 & 2.90 & 22.14 & 34.49 \\
8 & 3.38 & 2.77 & 29.99 & 75.69 \\
9 & 0.47 & 2.58 & 16.92 & 30.01 \\
10 & 4.90 & 3.83 & 33.98 & 64.76 \\
11 & 3.33 & 3.69 & 34.30 & 62.85 \\
12 & 1.83 & 3.28 & 28.65 & 42.03 \\
13 & 0.98 & 2.81 & 21.39 & 85.85 \\
14 & 2.67 & 3.14 & 28.10 & 11.46 \\
15 & 2.15 & 2.97 & 26.61 & 11.89 \\
16 & 6.57 & 3.33 & 33.40 & 49.73 \\
17 & 7.60 & 3.50 & 33.78 & 68.75 \\
18 & 3.88 & 2.95 & nd & 11.13 \\
19 & 3.10 & 3.21 & 29.78 & 54.04 \\
20 & 2.21 & 3.23 & 27.84 & 26.44 \\
21 & 3.96 & 3.24 & 30.37 & 61.70
\end{tabular}

Interestingly, the calculated $\mathrm{PPB} \%$ do not correlate with experimentally determined HSA affinities. This finding suggests that PreADMET software is not suitable for prediction of interactions between plasma proteins and analyzed isoxazolone derivatives. The lack of correlation also indicates that experimental methods are required to characterize drug candidates and that they are superior to theoretical approaches. Similar observations were reported for the evaluation of other properties of drug candidates such as lipophilicity [21,22].

Subsequent step of our examination pertained to assessing the influence of lipophilicity on HSA binding affinity for of the investigated molecules. Generally, interactions between xenobiotics and HSA are non-specific in nature, however it is well established that molecular hydrophobicity is the dominant factor [4]. In spite of that, drug-plasma protein interactions have complex character including electron and spherical forces that impose also noticeable effects on PPB [8]. In the case of the investigated compounds some trends have been shown in Supplementary Figure S1. Nevertheless, even upon exclusion of the outliners, i.e., derivatives 1,2 , and 4 , from the analysis as indicated by the 2.5 sigma-rule, the regression coefficient $(R)$ is relatively low $(R=0.697)$ [23].

Generally, comparative studies performed by the Valko and co-workers showed that for neutral and basic drugs affinity to HSA can be plotted as a function of $\mathrm{CHI}_{\text {IAM }}$ indices [19]. Hence, taking into account that interactions that govern membrane and HSA binding are very similar [20], the retention data derived from IAM chromatography have been employed for estimation of HSA affinity. Both IAM chromatography experiments as well as molecular mechanism governing affinity to phospholipids for isoxazolone derivatives have been described in our previous publications [14]. Considering the fact that the investigated isoxazolones are preset in neutral form under physiological $\mathrm{pH}$ conditions, it can be assumed that there should be a relationship between the previously determined $\mathrm{CHI}_{\mathrm{IAM}}$ retention values and currently established $\log k_{\mathrm{HSA}}$ parameters. A linear relationship between $\mathrm{CHI}_{\mathrm{IAM}}$ and $\log k_{\mathrm{HSA}}$ was found $(R=0.834$; regression plot is on Supplementary Figure S2). On account of this finding, it has been confirmed that IAM chromatography can be used for prediction of HSA binding for the examined class of chemical entities. 
In order to get insight into molecular mechanisms that condition affinity of isoxazolones to HSA a QSRR analysis has been performed. Briefly, the QSRR approach links as mathematic formula the retention parameters assessed during chromatographic experiments with molecular descriptors and quantitatively differentiate solutes. In order to better understand the influence of isoxazolone structure on affinity to HSA, two QSRR models have been proposed. The first assumption links experimental and computational data whereas the second approach is based solely on theoretical descriptors. The MLR approach was chosen among the regression methods since MLR models are easy to interpret and directly relate to the original data. The stepwise regression mode of MLR has been chosen. Molecular descriptors subsequently introduced to MLR models are listed together with basic statistical parameters in Supplementary Table S3. Considering relatively small size of the investigated library of izoxazolones derivatives, the obtained model has been validated using leave-one-out procedures. The calculated descriptors, together with their full names, used for QSRR modeling are listed in Table 2, whereas the obtained multiple linear regression equations along with statistical figures are listed below as Equations (2) and (3):

$\log k_{\mathrm{HSA}}=0.168( \pm 0.027) \mathrm{CHI}_{\mathrm{IAM}}+0.826( \pm 0.143) \mathrm{CATS} 3 \mathrm{D} \_08 \_\mathrm{AL}+3.454( \pm 1.090) \mathrm{MATS} 6 \mathrm{v}-1.780( \pm 0.735)$

$$
\begin{gathered}
R=0.960 R^{2}=0.922 Q^{2}=0.858 \mathrm{~s}=0.642 \mathrm{p}==5 \times 10^{-8} \mathrm{n}=19 \\
\operatorname{RMSECV}=0.749 \operatorname{RMSECV}(\%)=27.78 \% \\
\log k_{\mathrm{HSA}}=0.866( \pm 0.160) \text { CATS3D_08_AL }+307.871( \pm 0.160) \mathrm{R} 8 \mathrm{v}+-1.084( \pm 0.293) \text { F03[C-N] } \\
-117.012( \pm 43.598) J \operatorname{SI} 5+10.890( \pm 3.035) \\
R=0.929 R^{2}=0.863 Q^{2}=0.696 \mathrm{~s}=0.800 \mathrm{p}=9 \times 10^{-7} \mathrm{n}=21 \\
\operatorname{RMSECV}=0.964 \operatorname{RMSECV}(\%)=33.43 \%
\end{gathered}
$$

Table 2. Molecular descriptors calculated by the Dragon 7.0 program

\begin{tabular}{cccccc}
\hline No & CATS3D_08_AL & R8v+ & F03[C-N] & JGI5 & MATS6v \\
\hline 1 & 0 & 0.004 & 6 & 0.028 & -0.012 \\
2 & 0 & 0.012 & 9 & 0.027 & 0.007 \\
3 & 0 & 0.009 & 7 & 0.038 & -0.147 \\
4 & 0 & 0.012 & 9 & 0.028 & -0.055 \\
5 & 0 & 0.013 & 7 & 0.028 & 0.008 \\
6 & 0 & 0.003 & 5 & 0.045 & -0.120 \\
7 & 0 & 0.003 & 5 & 0.044 & -0.330 \\
8 & 0 & 0.012 & 7 & 0.032 & 0.097 \\
9 & 0 & 0.001 & 5 & 0.043 & -0.384 \\
10 & 3 & 0.011 & 7 & 0.031 & -0.193 \\
11 & 0 & 0.011 & 6 & 0.038 & -0.263 \\
12 & 0 & 0.006 & 6 & 0.045 & -0.327 \\
13 & 0 & 0.007 & 6 & 0.045 & -0.111 \\
14 & 0 & 0.012 & 7 & 0.034 & -0.115 \\
15 & 0 & 0.011 & 7 & 0.034 & -0.058 \\
16 & 2 & 0.019 & 7 & 0.034 & 0.051 \\
17 & 4 & 0.010 & 7 & 0.032 & -0.172 \\
18 & 0 & 0.010 & 6 & 0.038 & 0.029 \\
19 & 2 & 0.010 & 7 & 0.034 & -0.339 \\
20 & 0 & 0.011 & 7 & 0.043 & -0.080 \\
21 & 2 & 0.011 & 7 & 0.034 & -0.006 \\
\hline
\end{tabular}

CATS3D_08_AL-CATS3D Acceptor-Lipophilic BIN 08 (8.000-9.000 Å); R8v+—R maximal autocorrelation of lag 8 / weighted by van der Waals volume; F03[C-N] — Frequency of C-N at topological distance 3; JGI5-mean topological charge index of order 5; MATS6v -Moran autocorrelation of lag 6 weighted by van der Waals volume.

The developed models met the Tropsha et al. criteria $R^{2}>0.6$ and $Q^{2}>0.5$ [22]. Additionally, RMSECV has been calculated, which importance was indicated in studies 
performed by Lučić [23], and expressed as absolute value and percentage as recommended for QSRR models by Taraji and co-workers [24]. The plots of the observed and predicted $\log k_{\mathrm{HSA}}$ parameters are presented in Figure 3.
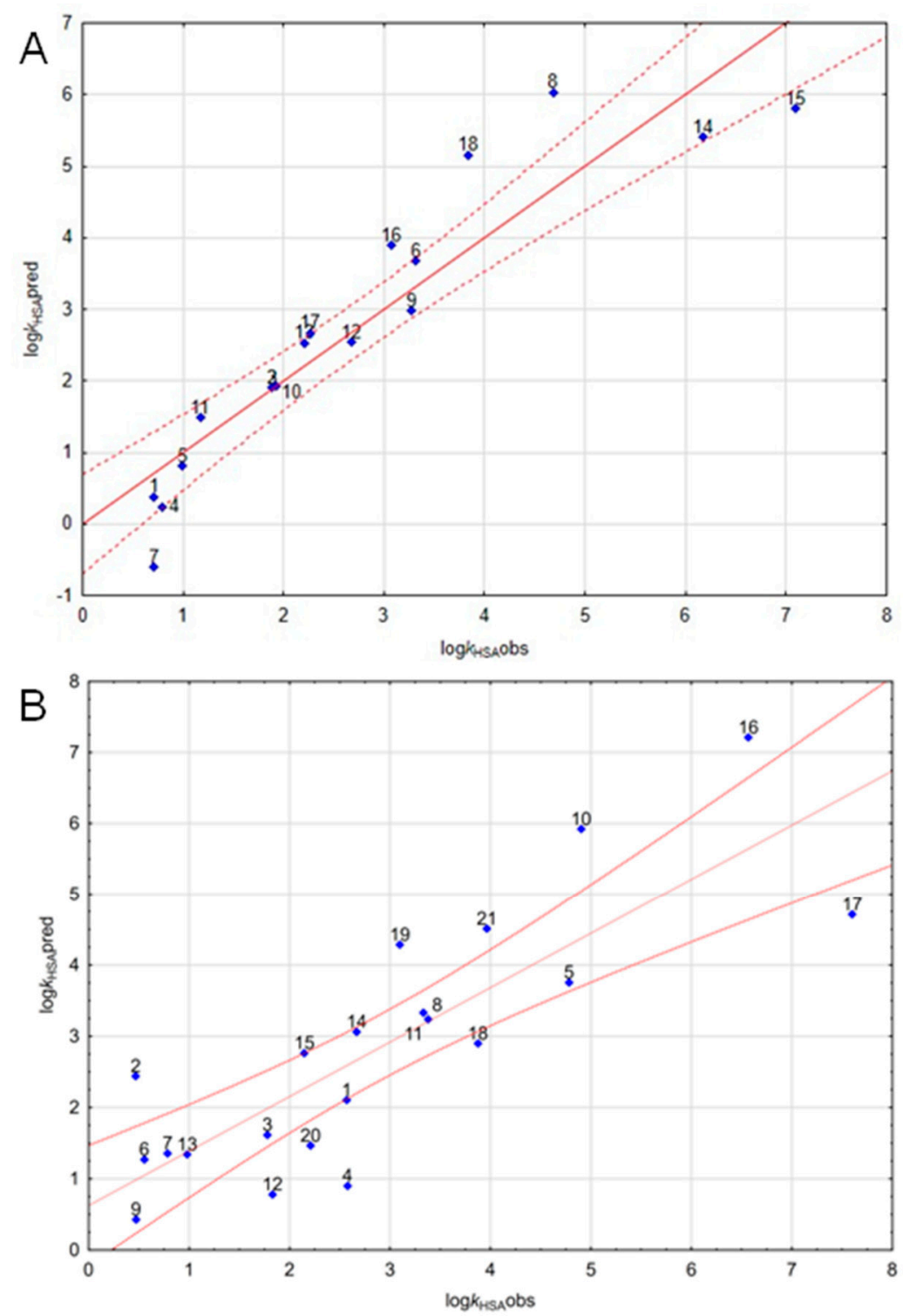

Figure 3. Comparison of experimentally determined $\log k_{\mathrm{HSA}}$ values and the corresponding parameters predicted by MLR-QSRR models: (A) model 1, (B) model 2.

The first model comprises experimentally determined $\mathrm{CHI}_{\mathrm{IAM}}$ indices as well as theoretical descriptors including CATS3D_08_AL and MATS6v, i.e., descriptors that pertain to lipophilicity of molecules $\left(\mathrm{CHI}_{\text {IAM }}\right.$; CATS3D_08_AL) and van der Waals volume (MATS6v).

The second model is based only theoretical descriptors. Among selected descriptors the highest weight has CATS3D_08_AL descriptor, which belong to chemically advanced template search (CATS) set of descriptors [25]. This group of parameters was introduced for the prediction of drug-receptor interactions, however our previous study proved its usefulness also for estimation of drug-HSA interactions which are of similar nature[26]. The applied CATS descriptor (CATS3D_08_AL) codes information regarding frequency of lipophilic (L) and hydrogen-bond acceptor (A) pairs of pharmacophore points corrected by topological properties of molecules. Subsequent descriptors utilized for model construction include data concerning van der Waals volume $(\mathrm{R} 8 \mathrm{v}+)$, topological charge index (JGI5), and frequency of $\mathrm{C}-\mathrm{N}$ atom pairs. 
Although, the calculated RMSECV is relatively high, it should be emphasized that this value is significantly affected by inclusion of compound 17. This molecule features the highest HSA-affinity among tested compounds, while the predicted value of the $\log k_{\text {HSA }}$ parameter is noticeably underestimated in both QSAR models. Taking into account the conducted experiments, it should be emphasized that the investigated isoxazolone derivatives display very diversified affinities to HSA, ranging from compounds that bind weakly to these proteins to congeners of very strong binding affinities $\left(\log k_{\mathrm{HSA}}\right.$ ranges between 0.47 to 7.60 ). Among the compounds tested, derivatives showing lowest affinities to HSA feature pyridine core, e.g., molecules 6 and 9. Also weak affinity to HSA displayed 1-benzyl derivative of quinolino-isoxazolone 2. Contrarily, the highest affinities to HSA showed 6-chloro- (16) and 1,6,7 trimethyl quinoline (17) derivatives with $\log k_{\mathrm{HSA}}$ values of 6.57 and 7.60, respectively. Since molecule 13 of promising antifungal activity proved relatively weak affinity to HSA $\left(\log k_{\mathrm{HSA}}=0.980\right)$, this structure can be considered as a starting point for the further development of antifungal isoxazolones.

\section{Conclusions}

The obtained QSRR models indicate that affinity of isoxazolone derivatives to HSA not only results from their lipophilicity but also rely upon on lipophilic centers, hydrogen-bond acceptors, as well as van der Waals volume. Finally, the results obtained indicate that retention parameters in $\mathrm{CHI}_{\mathrm{IAM}}$ and HAS chromatographic experiments correlate, while standard $\mathrm{C}_{18}$ separations and PreADMET software are not suitable for the assessment of HSA-isoxazolone interactions. The best alternative to HSA-HPLC experiments is the QSAR model that combines IAM chromatography retention parameters and theoretical descriptors.

Supplementary Materials: The following are available online at https:/ /www.mdpi.com/2227-971 7/9/3/512/s1. Figure S1: Comparison of $\log k_{\mathrm{W}}$ and $\log k_{\mathrm{HSA}}$ parameters determined with HPLC methods, Figure S2: Scatterplot comparing $\mathrm{CHI}_{\mathrm{IAM}}$ and logkHSA parameters, Table S1: Chemical names and 2D structures of pyridino- and quinolino-isoxazolone derivatives, Table S2: Retention times of the studied solutes measured by means of HSA-HPLC chromatography, Table S3: Statistical parameters of consecutive QSAR models calculated by progressive stepwise regression.

Author Contributions: Conceptualization, K.C.; methodology, K.C., M.P. and J.F.; software, K.C.; validation, H.K.; formal analysis, K.C. and J.F.; investigation, H.K.; resources, J.F. and J.S.; data curation, K.C.; writing-original draft preparation, K.C. and J.F.; writing-review and editing, J.S.; visualization, K.C. and J.F.; supervision, K.C.; project administration, K.C. and W.S.; funding acquisition, W.S. All authors have read and agreed to the published version of the manuscript.

Funding: This research was funded by the Ministry of Science and Higher Education by means of ST3 02-0003/07/518 statutory funds.

Institutional Review Board Statement: Not applicable.

Informed Consent Statement: Not applicable.

Data Availability Statement: The data presented in this study are available on request from the authors.

Conflicts of Interest: The authors declare no conflict of interest. The funders had no role in the design of the study; in the collection, analyses, or interpretation of data; in the writing of the manuscript, or in the decision to publish the results.

\section{References}

1. Wong, S.S.W.; Samaranayake, L.P.; Seneviratne, C.J. In pursuit of the ideal antifungal agent for Candida infections: Highthroughput screening of small molecules. Drug Discov. Today 2014, 19, 1721-1730. [CrossRef] [PubMed]

2. Vallabhaneni, S.; Mody, R.K.; Walker, T.; Chiller, T. The Global Burden of Fungal Diseases. Infect. Dis. Clin. N. Am. 2016, 30, 1-11. [CrossRef] [PubMed]

3. Seneviratne, C.J.; Rosa, E.A.R. Editorial: Antifungal drug discovery: New theories and new therapies. Front. Microbiol. 2016, 7 , 728. [CrossRef] [PubMed] 
4. Sanglard, D.; Coste, A.; Ferrari, S. Antifungal drug resistance mechanisms in fungal pathogens from the perspective of transcriptional gene regulation. FEMS Yeast Res. 2009, 9, 1029-1050. [CrossRef]

5. Clancy, C.J.; Nguyen, M.H. At what cost echinocandin resistance? J. Infect. Dis. 2011, 204, 499-501. [CrossRef]

6. Seneviratne, C.J.; Jin, L.J.; Samaranayake, Y.H.; Samaranayake, L.P. Cell density and cell aging as factors modulating antifungal resistance of Candida albicans biofilms. Antimicrob. Agents Chemother 2008, 52, 3259-3266. [CrossRef] [PubMed]

7. Stone, N.R.H.; Bicanic, T.; Salim, R.; Hope, W. Liposomal Amphotericin B (AmBisome ${ }^{\circledR}$ ): A Review of the Pharmacokinetics, Pharmacodynamics, Clinical Experience and Future Directions. Drugs 2016, 76, 485-500. [CrossRef] [PubMed]

8. Wanat, K.; Brzezinska, E.; Sobanska, A. Aspects of Drug-Protein Binding and Methods of Analyzing the Phenomenon. Curr. Pharm. Des. 2018, 24, 2974-2985. [CrossRef]

9. Lambrinidis, G.; Vallianatou, T.; Tsantili-Kakoulidou, A. In vitro, in silico and integrated strategies for the estimation of plasma protein binding. Rev. Adv. Drug Deliv. Rev. 2015, 23, 27-45. [CrossRef]

10. Zsila, F. Subdomain IB is the third major drug binding region of human serum albumin: Toward the three-sites model. Mol. Pharm. 2013, 10, 1668-1682. [CrossRef]

11. Saczewski, J.; Fedorowicz, J.; Kedzia, A.; Ziolkowska-Klinkosz, M.; Jalinska, A. Synthesis and Antifungal Activity of Some 4,6-Dimethylisoxazolo[3,4- b]pyridin-3(1H)-one Derivatives. Med. Chem. 2016, 12, 640-646. [CrossRef] [PubMed]

12. Saczewski, J.; Kedzia, A.; Jalińska, A. New derivatives of 4,6-dimethylisoxazolo[3,4-b]pyridin-3(1H)-one: Synthesis, tautomerism, electronic structure and antibacterial activity. Heterocycl. Commun. 2014, 20, 215. [CrossRef]

13. Saczewski, J.; Fedorowicz, J.; Korcz, M.; Saczewski, F.; Wicher, B.; Gdaniec, M.; Konopacka, A. Experimental and theoretical studies on the tautomerism and reactivity of isoxazolo[3,4-b]quinolin-3(1H)-ones. Tetrahedron 2015, 71, 8975-8984. [CrossRef]

14. Ciura, K.; Fedorowicz, J.; Žuvela, P.; Lovrić, M.; Kapica, H.; Baranowski, P.; Sawicki, W.; Wong, M.W.; Sączewski, J. Affinity of Antifungal Isoxazolo[3,4-b]pyridine-3(1H)-Ones to Phospholipids in Immobilized Artificial Membrane (IAM) Chromatography. Molecules 2020, 25, 4835. [CrossRef] [PubMed]

15. Ciura, K.; Fedorowicz, J.; Andrić, F.; Žuvela, P.; Greber, K.E.; Baranowski, P.; Kawczak, P.; Nowakowska, J.; Baçzek, T.; Saçzewski, J. Lipophilicity determination of antifungal isoxazolo[3,4-b]pyridin-3(1H)-ones and their N1-substituted derivatives with chromatographic and computational methods. Molecules 2019, 24, 4311. [CrossRef]

16. Ernzerhof, M.; Scuseria, G.E. Assessment of the Perdew-Burke-Ernzerhof exchange-correlation functional. J. Chem. Phys. 1999, 110, 5029-5036. [CrossRef]

17. Marenich, A.V.; Cramer, C.J.; Truhlar, D.G. Universal solvation model based on solute electron density and on a continuum model of the solvent defined by the bulk dielectric constant and atomic surface tensions. J. Phys. Chem. B 2009, 113, 6378-6396. [CrossRef] [PubMed]

18. Valko, K.L. Application of biomimetic HPLC to estimate in vivo behavior of early drug discovery compounds. Futur. Drug Discov. 2019, 1, FDD11. [CrossRef]

19. Valko, K.; Nunhuck, S.; Bevan, C.; Abraham, M.H.; Reynolds, D.P. Fast Gradient HPLC Method to Determine Compounds Binding to Human Serum Albumin. Relationships with Octanol/Water and Immobilized Artificial Membrane Lipophilicity. J. Pharm. Sci. 2003, 92, 2236-2248. [CrossRef]

20. Hubicka, U.; Żuromska-Witek, B.; Komsta, Ł.; Krzek, J. Lipophilicity study of fifteen fluoroquinolones by reversed-phase thin-layer chromatography. Anal. Methods 2015, 7, 3841-3848. [CrossRef]

21. Dabrowska, M.; Starek, M.; Komsta, Ł.; Szafrański, P.; Stasiewicz-Urban, A.; Opoka, W. Assessment of the chromatographic lipophilicity of eight cephalosporins on different stationary phases. Eur. J. Pharm. Sci. 2017, 101, 115-124. [CrossRef] [PubMed]

22. Tropsha, A.; Gramatica, P.; Gombar, V. The Importance of Being Earnest: Validation is the Absolute Essential for Successful Application and Interpretation of QSPR Models. QSAR Comb. Sci. 2003, 22, 69-77. [CrossRef]

23. Lučić, B.; Batista, J.; Bojović, V.; Lovrić, M.; Kržić, A.S.; Bešlo, D.; Nadramija, D.; Vikić-Topić, D. Estimation of random accuracy and its use in validation of predictive quality of classification models within predictive challenges. Croat. Chem. Acta 2019, 92, 379-391. [CrossRef]

24. Taraji, M.; Haddad, P.R.; Amos, R.I.J.; Talebi, M.; Szucs, R.; Dolan, J.W.; Pohl, C.A. Error measures in quantitative structureretention relationships studies. J. Chromatogr. A 2017, 1524, 298-302. [CrossRef]

25. Reutlinger, M.; Koch, C.P.; Reker, D.; Todoroff, N.; Schneider, P.; Rodrigues, T.; Schneider, G. Chemically advanced template search (CATS) for scaffold-hopping and prospective target prediction for "orphan" molecules. Mol. Inform. 2013, 32, 133-138. [CrossRef] [PubMed]

26. Ciura, K.; Ulenberg, S.; Kapica, H.; Kawczak, P.; Belka, M.; Bączek, T. Drug affinity to human serum albumin prediction by retention of cetyltrimethylammonium bromide pseudostationary phase in micellar electrokinetic chromatography and chemically advanced template search descriptors. J. Pharm. Biomed. Anal. 2020, 188, 113423. [CrossRef] 